\title{
Gender- and age-related differences in clinical presentation and management of outpatients with stable coronary artery disease
}

\author{
Roberto Ferrari ${ }^{a}$, Hélène Abergel ${ }^{b}$, lan Ford ${ }^{c}$, Kim M. Fox $^{d}$, Nicola Greenlaw ${ }^{c}$, \\ Ph.Gabriel Steg ${ }^{b}$, Dayi Hu ${ }^{e}$, Michael Tendera ${ }^{f}$, Jean-Claude Tardif ${ }^{9}$; for the CLARIFY \\ Investigators
}

\author{
a Department of Cardiology and LTTA Centre, University of Ferrara, Salvatore Maugeri \\ Foundation, IRCCS, Lumezzan, Italy \\ b INSERM U698, F75018 Paris, France; Univ Paris-Diderot, Sorbonne Paris Cité, Paris, France; \\ AP-HP, Hôpital Bichat, F75018, Paris, France \\ c Robertson Centre for Biostatistics, University of Glasgow, Glasgow, UK \\ d NHLI Imperial College, ICMS, Royal Brompton Hospital, London, UK \\ e Chongqing Medical University, Chongqing, China \\ f Medical University of Silesia, Katowice, Poland \\ g Montreal Heart Institute, Université de Montreal, Montreal, Canada \\ Correspondence: \\ Professor Roberto Ferrari, MD, PhD \\ Department of Cardiology, University of Ferrara, \\ Arcispedale S. Anna, Corso Giovecca 203 \\ 44100 Ferrara, Italy \\ Tel: +390532242011 \\ Fax: +39 0532241885 \\ E-mail: fri@unife.it
}

Word count: Abstract (236), Text (2559), References (30), Tables (2), Figures (2) 


\section{ABSTRACT (236 words)}

Introduction: Contemporary generalizable data on the demographics and management of outpatients with stable coronary artery disease (CAD) in routine clinical practice are sparse. Using data from the CLARIFY registry we describe gender- and age-related differences in baseline characteristics and management of these patients across broad geographic regions.

Methods: This international, prospective, observational, longitudinal registry, enrolled stable CAD outpatients from 45 countries in Africa, Asia, Australia, Europe, the Middle East, and North, Central and South America.

Results: Baseline data were available for 33,280 patients. Mean (SD) age was 64 (10.5) years and $22.5 \%$ of patients were female. The prevalence of CAD risk factors was generally higher in women than men. Women were older (66.6 vs 63.4 years), more frequently diagnosed with diabetes (33\% vs $28 \%$ ), hypertension (79\% vs $69 \%$ ), and higher resting heart rate (69 vs 67 bpm), and were less physically active. Smoking and a history of myocardial infarction were more common in men. Women were more likely to have angina (28\% vs $20 \%$ ), but less likely to have undergone revascularization procedures. CAD was more likely to be asymptomatic in older patients perhaps because of reduced levels of physical activity. Prescription of evidence-based medication for secondary prevention varied with age, with patients $\geq 75$ years less often treated with beta blockers, aspirin and angiotensin-converting enzyme inhibitors than patients <65 years.

Conclusions: Important gender-related differences in clinical characteristics and management continue to exist in all age groups of outpatients with stable CAD.

Keywords:

Angina; Coronary artery disease; Gender; Age 


\section{Introduction}

Average life expectancy has increased by 10 years in the last 30 years with improvements in the field of cardiology contributing for at least 7 years [1]. Despite such success, coronary artery disease (CAD) remains the leading cause of mortality and morbidity in the world [2-4]. It follows that the cardiovascular community has not solved the problem, but has delayed the occurrence of CAD-related mortality and changed the demographics of patients with CAD, with a shift of disease burden toward older patients, in both men and women $[5,6]$.

Aging has been associated with a reduced likelihood of receiving effective cardiac therapies [7-10]. Furthermore, the prevention and treatment of CAD may not be as effective in women as in men [11-18], suggesting that for optimal treatment of CAD it is necessary to recognize the impact of age and gender differences on the outcomes of care.

Surprisingly, there is a paucity of contemporary data regarding the clinical characteristics and management of outpatients with stable CAD. The majority of data are restricted to a particular manifestation of disease such as angina or acute coronary syndrome [6, 14, 19-24] and are limited to a specific country or a geographic area. Furthermore, gender and age are usually analyzed separately [21, 22, 24-26]. Our goal is to describe the gender- and age-based differences in baseline characteristics and management of outpatients with stable CAD using a large contemporary database from 45 countries.

\section{Methods}

\subsection{Study design}

CLARIFY is an ongoing international, prospective, observational, longitudinal cohort study in stable CAD outpatients with a 5-year follow-up [27]. Patients were enrolled in 45 countries in Africa, Asia, Australia, Europe, the Middle East, and North, Central and South America. They were treated according to usual clinical practice at each institution, with no specific tests or therapies defined in the study protocol. CLARIFY is registered in the ISRCTN registry of clinical trials with the number ISRCTN43070564 and approved 
by local ethical committees. All subjects provided written informed consent in accordance with national and local guidelines.

\subsection{Study population}

Outpatients with stable CAD had to meet at least one of the following criteria to be eligible for enrollment [27]: documented myocardial infarction > 3 months previously; at least one angiographically proved $>50 \%$ diameter coronary artery stenosis; chest pain with myocardial ischemia proven by stress echocardiography (ECG), or myocardial imaging; and history of coronary artery bypass graft (CABG) or percutaneous coronary intervention $(\mathrm{PCl})$ carried out $>3$ months previously. Patients were not eligible if they had been hospitalized for cardiovascular disease (CVD) within the last 3 months (including revascularization), or had planned revascularization, or had conditions hampering participation or 5-year follow-up such as limited cooperation or legal capacity, advanced heart failure, severe valve disease, history of valve repair/replacement, or serious non-cardiovascular disease limiting life expectancy (cancer, drug abuse, etc.),

Patients were recruited between November 2009 and July 2010. To ensure a balanced representation of participating countries, a general target enrollment for any participating country was 25 patients per million inhabitants (range 12.5-50). A broad range of physicians were involved in the selection of patients including cardiologists, general practitioners and internists. Each physician recruited a maximum of 15 outpatients with stable CAD as defined by the protocol. Patients were enrolled in each practice over a brief period of time to provide near, if not consecutive, patient enrollment.

\subsection{Baseline data collection and data management}

Data regarding patient demographics, medical history, risk factors, employment status, physical examination, heart rate (determined by both pulse palpation and 12-lead electrocardiography) using the most recent ECG within 6 months in clinically stable patients, laboratory values (if available), and current chronic medical treatments were collected by the participating physician using an electronic case report form and sent to the data management center. Here, data were checked for completeness and internal consistency. Data quality control was performed onsite in $5 \%$ of sites chosen at random 
in each country. At each of these sites, $100 \%$ of case report forms for patients enrolled at that site were monitored for source documentation and accuracy.

\subsection{Statistical analysis}

Data were collected and analyzed by an independent statistics center, the Robertson Center for Biostatistics, University of Glasgow, UK. Continuous variables are presented as means and standard deviations (SDs) in case of normal distribution, or medians and interquartile ranges (IQRs) and ranges in case of non-normal distribution. Categorical data are presented as counts and percentages.

\section{Results}

The CLARIFY registry screened a total of 33432 patients from 45 countries. Baseline data were available for 33 280; 47 patients did not meet the inclusion criteria and 105 did not provide consent.

The mean (SD) age of the population surveyed was 64 (10.5) years. Women represented $22.5 \%$ of the population overall and were slightly older than the men with a mean age of 66.6 (10.0) years compared with 63.4 (10.5) years in men. A total of 17206 patients were $<65$ years old, 10812 were aged $65-74$ years, and 5196 were aged $\geq 75$ years.

\subsection{Gender differences in the presentation and clinical management of outpatients with} $C A D$

The baseline data for men and women are presented in Tables 1 and 2. A number of important differences in clinical characteristics and management were identified. Compared with men, women were on average 3.1 years older and had higher resting heart rates (69.0 bpm versus $67.9 \mathrm{bpm}$, pulse palpation) and systolic blood pressure (133.3 $\mathrm{mmHg}$ versus $130.3 \mathrm{mmHg}$ ). Women were less physically active with $23.4 \%$ performing no physical activity compared with $14.3 \%$ of men. Only $9.6 \%$ of women performed at least 20 minutes of vigorous physical activity at least three times per week 
compared with $17.3 \%$ of men, and only $11.9 \%$ of women versus $18.2 \%$ of men performed such activity once or twice per week.

In terms of cardiovascular history, women had a shorter duration of CAD (4 years versus 5 years, $P<0.0001$ ) and were more frequently diagnosed with diabetes, hypertension, stroke, and transient ischemic attack. Smoking, a history of myocardial infarction, and peripheral arterial disease (PAD) were more common in men. Women presented with angina more often than men (28\% versus $20 \%)$ and this was true for all angina classes defined by the Canadian Cardiovascular Society grading scale (Fig. 1). In contrast, women were less likely to have undergone $\mathrm{PCl}(54.9 \%$ versus $59.8 \%)$ or CABG $(17.8 \%$ versus $25.0 \%$ ) (Fig. 2).

Women were less likely to have undergone cardiac investigations. Coronary angiography was performed in $79.9 \%$ of women versus $87.0 \%$ of men. Noninvasive tests for myocardial ischemia were done in $58.3 \%$ of women compared with $62.9 \%$ in men. In those patients with data from noninvasive testing, evidence for myocardial ischemia was observed more often in women than men (17.3\% vs $15.9 \%)$.

Women were prescribed angiotensin-converting enzyme (ACE) inhibitors and lipidlowering drugs significantly less frequently than men, but received antianginal agents, antidiabetes drugs, non-steroidal anti-inflammatory, proton pump inhibitor, and thyroid hormone replacement more often than men (Fig. 2). There was no significant difference in the use of beta-blockers between the sexes.

\subsection{Age-related differences in the presentation and clinical management of outpatients} with $C A D$

Table 1 demonstrates that concomitant conditions were widely prevalent among the study population. The proportion of those with atrial fibrillation, transient ischemic attack, hospitalization for congestive heart failure, asthma/chronic obstructive pulmonary disease (COPD), PAD, dyslipidemia, hypertension, and diabetes increased progressively with age. Tobacco and alcohol use decreased relative to age.

There were significant age-related differences among both men and women (Table 1). Compared with those aged $<65$ years, patients aged $\geq 75$ years had higher systolic blood pressure $(133.55$ [16.97] $\mathrm{mmHg}$ versus 129.38 [16.47] [values were the same in both 
men and women]), lower body mass index (BMl) values $\left(26.1 \mathrm{~kg} / \mathrm{m}^{2}\right.$ versus $27.9 \mathrm{~kg} / \mathrm{m}^{2}$ in women and $26.5 \mathrm{~kg} / \mathrm{m}^{2}$ versus $27.6 \mathrm{~kg} / \mathrm{m}^{2}$ in men) and were less likely to be current smokers (2.3\% versus $11.5 \%$ in women and $4.5 \%$ versus $19.6 \%$ in men). Patients $\geq 75$ years had lower resting heart rates measured by pulse palpation ( $67.5 \mathrm{bpm}$ versus 69.1 bpm) or by ECG (66.6 bpm versus $67.8 \mathrm{bpm}$ ) (values were the same in both men and women).

In both men and women, older patients were more often asymptomatic, with angina present in $20.7 \%$ of women and $17.1 \%$ of men aged $\geq 75$ years compared with $32.7 \%$ of women and $22.9 \%$ of men aged $<65$ years $(P<0.0001)$. This may be due to reduced physical activity below the ischemic threshold, sedentary lifestyle or locomotor disability. Nearly one third of women (31.3\%) and $21.9 \%$ of men aged $\geq 75$ years performed no physical activity compared with $19.8 \%$ of women and $12.2 \%$ of men aged $<65$ years $(P<0.0001)$.

Older patients had significantly higher rates of hypertension $(74.7 \%$ versus $64.7 \%$ in men and $82.8 \%$ versus $73.2 \%$ in women), PAD (14.7\% versus $7.6 \%$ in men and $9.9 \%$ versus $6.8 \%$ in women), hospitalization for chronic heart failure $(7.1 \%$ versus $3.6 \%$ in men and $8.3 \%$ versus $4.4 \%$ in women), asthma or COPD $(11.0 \%$ versus $5.0 \%$ in men and $9.1 \%$ versus $7.6 \%$ in women), and stroke (6.4\% versus $2.7 \%$ in men and $5.8 \%$ versus $4.1 \%$ in women). Older men had a higher rate of diabetes ( $28.8 \%$ versus $26.7 \%$ ); in women, there was no significant difference in rates of diabetes with age.

Men of all ages were significantly more likely to have undergone CABG than women (Table 1). In patients $<65$ years of age percutaneous coronary interventions were more common in men than women, but in older patients the proportion of men and women having these interventions was similar.

Table 2 shows the principal evidence-based medications prescribed across age strata. Compared with younger patients, those over 75 years were less often treated with betablockers $(68.4 \%$ versus $78.4 \%$ in men and $69.1 \%$ versus $77.9 \%$ in women), aspirin (79.2\% versus $91.5 \%$ in men and $82.8 \%$ versus $89.1 \%$ in women), and ACE inhibitors ( $47.0 \%$ versus $55.5 \%$ in men and $39.6 \%$ versus $49.7 \%$ in women), but received more often calcium channel blockers (31.7\% versus $22.0 \%$ in men and $34.1 \%$ versus $29.6 \%$ in women), angiotensin II receptor blockers $(29.0 \%$ versus $22.1 \%$ in men and $38.5 \%$ 
versus $27.3 \%$ in women), long-acting nitrates $(26.8 \%$ versus $19.1 \%$ in men and $30.1 \%$ versus $23.5 \%$ in women) and diuretics (37.6\% versus $21.7 \%$ in men and $44.5 \%$ versus $32.3 \%$ in women).

\section{Discussion}

Our analysis reveals a number of gender- and age-related differences among stable outpatients with CAD. These findings have important clinical implications. The positive impact of improvements in the prevention and treatment of CAD has resulted in an increased number of elderly patients of both genders living as outpatients and needing targeted care. In terms of risk factors, older patients have lower heart rates compared with those aged $<65$ years, but higher systolic blood pressure, a higher prevalence of diabetes, and are less likely to be current smokers. There were also significant differences in current medications used by different age groups. Increasing age was associated with a reduced likelihood of receiving effective cardiac therapies such as ACE inhibitors, lipid-lowering agents and beta-blockers, but more symptomatic therapy.

This paradox of less intensive use of evidence based preventive therapies in patients recognized to be at higher risk may reflect attempts by physicians to limit the number of cardiovascular medications in elderly patients who often need other drugs for comorbid conditions, or to the questionable belief that in elderly patients symptom relief is more important than prognosis.

A number of gender-related differences were also relevant. When compared with their age-matched male counterparts, women with CAD were older, less likely to be current smokers, but performed less or no physical activity. Women had more history of diabetes, high blood pressure and angina, yet were less likely than men to undergo coronary artery angiography as well as non-invasive tests for myocardial ischemia. This latter discrepancy is probably related to the rather low specificity of exercise testing in women. As a consequence, women are less likely than men to undergo PCI or CABG. Pharmacological therapy for CAD also differed between the sexes. Women were more likely to receive calcium channel blockers, but less ACE inhibitors, more anti-diabetic drugs, but less statins than men. There were no differences in the use of beta-blockers between men and women. 
Other studies have also shown that men are significantly more likely than women to undergo revascularisation with both $C A B G$ and $P C I[14,17,28]$. The reason for this discrepancy is unclear. Physicians appear to be less concerned about CAD in women as, despite the higher degree of angina, women received fewer referrals for coronary angiography. It is also possible that the perception of CAD is less in women than in men so that they consult their physicians less frequently. In the past, revascularisation procedures were associated with more complications in women than men, but technical advances in the last decade have greatly improved outcomes in women [29]. Other reasons for the discrepancy could be linked to differences in clinical judgment or to gender differences in the severity and extent of diseased coronary arteries. In the current study, men had more severe coronary stenoses than women, which may in part explain the lower rate of procedures in women. However, women were also less likely to be receiving recommended treatment with ACE inhibitors and lipid-lowering drugs than their male counterparts. The physician's perception of either anticipated adverse drug reactions or less severe disease may influence their decision to prescribe these medications for women [30]. When taken together, these data suggest the need to increase awareness of CAD in women both among physicians and the female population.

Other studies have reported similar findings [7, 9, 11-12, 14, 17, 25]. However, many of these were completed over a decade ago and may not reflect current clinical practice. Furthermore, many have focused on specific subgroups of patients with a particular manifestation of disease such as stable angina or acute coronary syndromes. Others focused on populations from randomized clinical trials with their more narrowly defined inclusion/exclusion criteria. The CLARIFY data are an important addition to the literature as they provide robust contemporary information about the characteristics, management, and outcomes of a large population of stable CAD outpatients across a variety of geographic and practice settings. Furthermore, the entire clinical management pathway of patients with stable CAD was examined, rather than restricting the study to a limited number of healthcare interventions.

'Real-life' studies such as CLARIFY offer the advantage that they provide data on a heterogeneous patient population that includes groups who are often under-represented in randomized clinical trials. However, as a non-randomized, observational study, CLARIFY is subject to certain limitations and potential biases. We did not collect 
information about the adverse effects related to the use of medications, or reasons by patients as well as physicians for not using the medications or undergoing cardiac interventions. Finally, we did not examine the effect of potential confounders when evaluating gender- and age-differences in patients' characteristics and CAD management. However, despite these limitations, the study provides a useful insight into current practice with regard to the characteristics and management of outpatients with stable CAD.

The CLARIFY data reveal that despite the attention that has been focused on gender and age-related differences over the past 20 years, the disparities have remained largely unchanged over time. Women and the elderly form a major part of the population that characterizes contemporary outpatients with stable CAD, yet, despite appearing at higher risk of subsequent events, based on increased prevalence of risk factors and more frequent history of $\mathrm{CV}$ events, they receive less intensive preventive evidence based therapies. In high risk patients, particularly those with established CAD, vigorous preventive measures are warranted at all ages and in both sexes. Further work is required to improve our understanding of the age and gender differences in patient characteristics and CAD management identified in the CLARIFY registry. 


\section{References}

1. Cutler D, McClellan M. Is Technological Change in Medicine Worth It?" Health Affairs. 2001;20:11-29

2. British Heart Foundation. Coronary Heart Disease Statistics 2010.

http//www.heartstats.org. Last accessed January 2012.

3. World Health Organization. Global atlas on cardiovascular disease prevention and control. Mendis S, Puska P, Norrving B, editors. World Health Organization, Geneva 2011.

4. Roger VL, Go AS, Lloyd-Jones DM, et al; on behalf of the American Heart Association Statistics Committee and Stroke Statistics Subcommittee. Heart Disease and Stroke Statistics-2011 Update. A Report From the American Heart Association. Circulation 2011;123:e18-e209.

5. Mathers CD, Loncar D. Projections of global mortality and burden of disease from 2002 to 2030. PLoS Med 2006;3:e442.

6. Mannsverk J, Wilsgaard T, Njølstad I, et al. Age and gender differences in incidence and case fatality trends for myocardial infarction: a 30-year follow-up. The Tromso Study. Eur J Cardiovasc Prev Rehabil 2011; Aug 22. [Epub ahead of print].

7. Harrold LR, Lessard D, Yarzebski J, Gurwitz JH, Gore JM, Goldberg RJ. Age and sex differences in the treatment of patients with initial acute myocardial infarction: a community-wide perspective. Cardiology 2003;99:39-46.

8. McLaughlin TJ, Soumerai SB, Willison DJ, et al. Adherence to national guidelines for drug treatment of suspected acute myocardial infarction: evidence for undertreatment in women and the elderly. Arch Intern Med 1996;156:799-805.

9. Tran CT, Laupacis A, Mamdani MM, Tu JV. Effect of age on the use of evidencebased therapies for acute myocardial infarction. Am Heart J 2004;148:834-841. 
10. Vitale C, Spoletini I, Volterrani M, lellamo F, Fini M. Pattern of use of $\beta$-blockers in older patients with stable coronary artery disease: an observational, cross-sectional, multicentre survey. Drugs Aging 2011;28:703-11.

11. Maynard C, Litwin PE, Martin JS, Weaver WD. Gender differences in the treatment and outcome of acute myocardial infarction: results from the Myocardial Infarction Triage and Intervention Registry. Arch Intern Med 1992;152:972-976.

12. Kudenchuk PJ, Maynard C, Martin JS, Wirkus M, Weaver WD. Comparison of presentation, treatment, and outcome of acute myocardial infarction in men versus women (the Myocardial Infarction Triage and Intervention Registry). Am J Cardiol 1996;78:9-14.

13. Beaulieu MD, Blais R, Jacques A, Battista RN, Lebeau R, Brophy J. Are patients suffering from stable angina receiving optimal medical treatment? Q J Med 2001;94:301308.

14. Daly C, Clemens F, Lopez Sendon JL, et al; Euro Heart Survey Investigators. Gender differences in the management and clinical outcome of stable angina. Circulation 2006;113:490-498.

15. D’Antono B, Dupuis G, Fortin C, Arsenault A, Burelle D. Angina symptoms in men and women with stable coronary artery disease and evidence of exercise-induced myocardial perfusion defects. Am Heart J 2006;151:813-819.

16. Glaser R, Selzer F, Jacobs AK, et al. Effect of gender on prognosis following percutaneous coronary intervention for stable angina pectoris and acute coronary syndromes. Am J Cardiol 2006;98:1446-1150.

17. Crilly M, Bundred P, Hu X, Leckey L, Johnstone F. Gender differences in the clinical management of patients with angina pectoris: a cross-sectional survey in primary care. BMC Health Serv Res 2007;7:142.

18. Tavris D, Shoaibi A, Chen AY, Uchida T, Roe MT, Chen J. Gender differences in the treatment of non-ST-segment elevation myocardial infarction. Clin Cardiol 2010;33:99103. 
19. Lampert R, McPherson CA, Clancy JF, Caulin-Glaser TL, Rosenfeld LE, Batsford WP. Gender differences in ventricular arrhythmia recurrence in patients with coronary artery disease and implantable cardioverter-defibrillators. J Am Coll Cardiol 2004;43:2293-9.

20. Pepine CJ, Abrams J, Marks RG, Morris JJ, Scheidt SS, Handberg E. Characteristics of a contemporary population with angina pectoris. TIDES Investigators. Am J Cardiol 1994;74:226-231.

21. Shaw LJ, Shaw RE, Merz CN, et al; American College of Cardiology-National Cardiovascular Data Registry Investigators. Impact of ethnicity and gender differences on angiographic coronary artery disease prevalence and in-hospital mortality in the American College of Cardiology-National Cardiovascular Data Registry. Circulation 2008;117:1787-801.

22. Lahoz C, Mantilla T, Taboada M, et al. Gender differences in evidence-based pharmacological therapy for patients with stable coronary heart disease. Int $\mathrm{J}$ Cardiol 2009;133:336-340.

23. Reibis RK, Bestehorn K, Pittrow D, Jannowitz C, Wegscheider K, Völler H. Elevated risk profile of women in secondary prevention of coronary artery disease: a 6-year survey of 117,913 patients. J Womens Health (Larchmt) 2009;18:1123-1131.

24. Coppieters $Y$, Collart $P$, Levêque A. Gender differences in acute myocardial infarction, twenty-five years registration. Int J Cardiol 2011 May 6. [Epub ahead of print]

25. Gurjeva OS, Roe MT, Murphy SA, Moliterno DJ, Cannon CP; GUARANTEE investigators. Unfortunate impact of age on the management and outcomes of unstable angina and non-ST elevation myocardial infarction (The GUARANTEE Registry). Crit Pathw Cardiol 2005;4:81-87.

26. Ky B, Kirwan BA, de Brouwer S, et al. Gender differences in cardiac remodeling and clinical outcomes in chronic stable angina pectoris (from the ACTION Trial). Am J Cardiol 2010;105:943-947. 
27. Steg PG. Heart rate management in coronary artery disease: the CLARIFY registry. Eur Heart J Suppl 2009;11(Supple D):D13-D18.

28. Spencer I, Unwin N, Pledger G. Hospital investigation of men and women treated for angina. BMJ. 1995;310:1576.

29. Dunlay SM, Rihal CS, Sundt TM, Gerber Y, Roger VL. Current trends in coronary revascularization. Curr Treat Options Cardiovasc Med. 2009;11(1):61-70

30. Enriquez JR, Pratap P, Zbilut JP, Calvin JE, Volgman AS. Women tolerate drug therapy for coronary artery disease as well as men do, but are treated less frequently with aspirin, beta-blockers, or statins. Gend Med 2008;5:53-61. 
Table 1 Clinical characteristics and patient management of men and women in the CLARIFY registry.

Fig. 1. Distribution of Canadian Cardiovascular Society angina classes in men and women.

Fig. 2. Clinical characteristics and management of CAD patients by gender. 